\title{
Iran's Medical Tourism Development Model in Cardiac Surgery
}

\author{
Fatemeh Noori ${ }^{1}$, Mehrdad Kargari ${ }^{1, *}$ \\ ${ }^{1}$ Faculty of Systems and Industrial Engineering, Tarbiat Modares University, Tehran, IR Iran
}

*Corresponding Author: Mehrdad Kargari, Associate Professor, Faculty of Systems and Industrial Engineering, Tarbiat Modares University, Tehran, Iran. Tel: +98-21-82884955, Email: m_kargari@modares.ac.ir

\begin{abstract}
Introduction: Since medical tourism is considered as an incremental activity in this sector and proper infrastructures in country to make medical tourism are lacking, announcement by authorities to provide perquisites of medical tourism to make the first clinic hotel and health town are necessary for the purpose of developing medical tourism in Iran, all of these side issues should be investigated.

Methods: Cardiovascular diseases are very common because of pollution and industrial development. In this research, by extracting related studies on medical tourism and its localization, using hospitals data bank and questionnaire of 640 medical tourists, only 528 of them were approved and finally by using IBM SPSS modular 14.1 software decision tree in data algorithm, efficiency and purity level were obtained. The method for data preprocess step is utilized to extract the best model. Two preprocess steps are deleting useless and correlated features, because data should be prepared until model has the least error.

Results: Among the examined algorithm including C\&RT, CHAID and Multiple Linear Regression, it was shown that C\&RT has the optimal results. The results obtained from this research indicate that C\&RT binary decision tree has the smallest error value (0.078) and the greatest accuracy value (0.922).

Conclusion: In this research, first effective factors on heart medical tourism were investigated with the help of experts in this field, then C\&RT and CHASID models using Clementine software and multiple linear regression variables were compared and ranked. With respect to this algorithm, personnel behavior, social security and communication variables are respectively the most important factors for medical tourist attraction.
\end{abstract}

Keywords: Cardiac Surgery, Medical Information System, Medical Tourism

Article History: Received: 29 Jan. 2016; Accepted: 10 Apr. 2016; Online Published: 25 Jun. 2016

Cite this article as: Noori F, Kargari M. Iran's medical tourism development model in cardiac surgery. Int J Travel Med Glob Health. 2016;4(2):57-64. doi: 10.20286/ijtmgh-04025

\section{Introduction}

Since medical tourism is considered as an incremental activity in this sector and proper infrastructures in country to make medical tourism are lacking, announcement by authorities to provide perquisites of medical tourism to make the first clinic hotel and health town are necessary for the purpose of developing medical tourism in Iran, all of these side issues should be investigated [1-3].

On the other hand; although, Iran is among the ten tourism destination pioneers for its tourisms attractions, nature, climate, and historical places, it doesn't take significant part in world tourism. However, medical tourism is the third industry and some countries such as Spain earn living in this way. Today, medical tourism has increase in Iran and the world being industrial with the occurrence of different physical and mental illnesses and human interest on significant cosmetic surgery. Nevertheless, the concern of our country is whether this industry should blossom or be forgotten despite all facilities used for it $[4,5]$.

Four main factors should be considered in medical tourism and attracting medical tourist. Healthcare professional capabilities of employees, cutting-edge technologies and international standards, treatment and care-cost economy and domestic regulations are other important factors in medical tourism [6].

Iran is considered as the most powerful country in all four domains in the Middle East region and neighboring countries and this potential must be fully exploited. Unfortunately, there are no appropriate advertisements to show Iran medical abilities and tourism and governmental media and embassies have more significant role to play in this field [7]. Old background: The term "health" implies preventing, treating, and protecting the mental and physical well-being by providing professional nursing and medical services.
Medical abilities and tourism and governmental media and embassies have more significant role to play in this field [8]. This paper tries to confirm or reject these hypotheses that if it is possible to obtain a proper framework and strong foundation to implement and expand surveying industry of medical tourism in Iran by identifying the effective processes in medical tourism; medical tourism condition in Iran is precisely identifiable and analyzable in the present condition; the field of cardiovascular surgery in medical tourism is precisely identifiable and analyzable; proper suggestions and policies can be proposed to develop medical tourism industry; Canvas model can be proposed to implement medical tourism by the obtained reports; medical tourism can be predicted for patients by needed processes in medical centers. Since this research describes and studies the existing situation, its methodology is descriptive. Descriptive study is carried out to determine and describe features of an occasion $[9,10]$.

Method of data analysis is quantitative and is classified by obtained data and measures dispersion and expansion indexes which are quantitative.

Tourism is extensive and a huge incremental industry in the world economy and culture field. Tourism encompasses nearly all aspects of the society. Today, the importance of this industry and expansion is analyzed using various phenomena based on tourist' views [11].

Travel medicine is the branch of medicine that deals with the prevention and management of medical problems of international travelers [12]. It involves familiarization with each other's cultures and understanding the differences better $[6,13]$. Undoubtedly, all the world countries are close rivals in using social, cultural, benefits and especially higher rank of incomes and employment rate by optimizing this service and providing this industry in their subordinate country $[14,15]$. 


\subsection{Medical Tourism in Iran}

Medical tourism was proposed independently in Iran by combining cultural heritage organization and tourism and Iran traveling organization in 2004 and it is in the fourth development plan in Article 87 of the Ministry of Health, and Medical Education. It is responsible for providing the way for an effective presence in global markets and to meet the health and medical needs of the Islamic Republic of Iran in the center of commercial area of strategic policy framework. It also facilitates the introduction of capabilities, supply and marketing of medical education and health services and products, equipment, medical and pharmaceutical products in such a way that the amount of foreign exchange earned from the export of products and services equals $30 \%$ of foreign exchange costs of the health sector at the end of the previous year of the fourth plan. Iran is rank fifth in having cultural heritage, rank $10^{\text {th }}$ in climate and $57^{\text {th }}$ among the world in attracting tourists, and our medical tourism rank is negligible $[2,4,8]$.

\subsection{Medical Tourism in the Field of Cardiac Surgery}

Medical tourism is a structured travel to improve and regain physical and mental health [12]. The $20^{\text {th }}$ century saw a significant growth in expansion and increase in hotel and medical centers and hot springs. Undoubtedly, all global countries are in close competitiveness to use social, cultural, conditions and high share of income and employment by optimization of this services and providing industry in their subordinate country. For high number of cardiovascular patients in the country, cardiac surgery is more economical in Iran for foreigners, due to the fact that various countries believe on Iranian physicians and this surgery is carried out by master specialized surgeon from the beginning to the end. Since age is afflicted to cardiac diseases, applicants for cardiac surgery have increased the potential of tourism extension based on cardiac surgery [16].

Medical tourism has various definitions according to health and tourism researchers and authorities. Management of various fields and tourism organization defines tourism as using services such as mineral waters, landscapes, waterfalls, jungles, lugs, sun showers, sand therapy, herbal therapy, or medical interventions to improve the health and personal spirit in places far from tourist resident for more than $24 \mathrm{~h}$.

Of course, the aim of the Ministry of health is to create jobs for the majority of medical graduates in this area; however, medical tourism was not thriving but later in 2004, medical tourism became independent after the combination of cultural heritage organization and Iran traveling and tourism organization. Iran government supplies $30 \%$ of the medical needs of the country based on its new planning at the end of the fifth development plan by exporting goods, medical services and medical tourism. With this development of medical tourism, there will be various benefits such as employment and quality growth in medical and economic sectors and inflow of foreign exchange especially in tourism industry $[16,17]$.

Ahmed et al. [12] believed that Iran has natural resources and also skillful physicians and advance medical facilities to extend this industry and provide good conditions. Despite all these facilities, our country has reduce share of medical tourism and countries entered without any support have gotten scores and increased their market share.

\section{Methods}

\subsection{Statistical Population}

Data was collected from 5 cardiac patients medical tourists who were hospitalized and questionnaires were also distributed among them to investigate effective factor on medical tourism and more effective factors to make authorities draw more attention to this matter.

\subsection{Statistical Sample and Sampling Method}

Patients who filled the questionnaire were selected as statistical sample. Final part of this questionnaire includes surveying ideas about heart health tourism; questionnaire includes health tourism in Iran. Our method was descriptive which is a method of data collection. Patients were asked to answer some specific questions based on the purpose of the study.

Tourism makes these opportunities for humans to learn from each other besides enjoying nature and human wonders and familiarize with each other's cultures and understand the differences better [6,10]. Undoubtedly, all the world countries are close rivals in using social, cultural, benefits and especially higher rank of incomes and employment rate by optimizing this service and providing this industry in their subordinate country $[3,11]$.

\subsection{Data Resources and Collection Methods}

In this research, related library resources including theses, specialized books, articles, and Persian and Latin journals in internet were utilized. To obtain necessary statistical data, related patients information was needed and also a questionnaire was used. In this research, test reliability was calculated by Cronbach alpha coefficient. This method measures various features to calculate internal coordination of measuring instrument, Cronbach alpha standard was used to investigate questionnaire and a value of 0.803 was obtained which shows necessary standards. Necessary data for this research was prepared from hospital authorities. Excel software was used to store data.

Satisfaction variable that shows medical tourists satisfaction for Iran shows that it is a dependent variable and other variables (factors) as independent variables. Generally, based on the questionnaire, variables are classified as:

1. Patient information: (country, sex, marital status, career, Age, treatment, time reference, discharge)

2. Tourism survey on the health of the patient-related variables: (the equipment, technology, the quality of care, awareness, the cost of treatment, handling of the patient, number of employees, needs, behavior of staff, personnel access, physicians, electronic banking, notices, electronic communications, social security, quality, hygiene, tourist attractions, amenities, compliance, transport cost, satisfaction)

Data was collected in Excel software. Total number of obtained data before process was 640 records and after data processing, 528 records were considered as research data. That is, 390 records of questionnaire and 138 records from hospital data bank.

The method for data preprocess step was used to extract the best model. Two preprocess steps are deleting unnecessary and correlated features, because data should be prepared until model has the least error.

Thereafter, in order to investigate effective factors on medical tourism (health) in Iran, classification method was utilized to analyze data. To classify data, CLEMENTINE 12 software, and CHAID C\&R Tree were utilized. To evaluate the method used, it was compared to multiple linear regression method; that is, descriptive statistical subcollection. Satisfaction variable shows patient illnesses from 
medical centers and can be considered as an objective variable. Used materials are a sample of decision tree. Decision tree is a tree structure and each of its nodes indicates a test on a feature. Each tree indicates a resultant of a test and tree leaves show classifications.

\subsection{Decision Tree}

Decision tree is a powerful and string instrument and yet common for classification and prediction purposes. Attraction of basic tree methods is based on the fact that decision trees show regulations. Regulations can easily change into Persian or any other languages to be understandable. In addition, they can change into accessible language to data banks such as SQL or extract a group data.

Decision tree is also useful for efficient evaluation of the vision about existing relationship among many input variables for an objective variable. Since a decision tree of data screening and modeling are combined with each other, they are considered as initial powerful step in modeling process even when some other techniques are used to prepare initial model.

\subsubsection{Preprocesses}

Before modeling, a data pre-process is required. For this purpose, columns have characters using alternatives coding.

In variable related to sex, we put 1 for woman and 0 for man.

In column related to marital status, we put 0 for single and 1 for married.

In column related to other departments, instead of CCU, daycare, ICU, Post-Angio, VIP1, VIP2, emergency, inpatient, and babies, we substituted it with 1 to 9 .

In column related to Job, instead of Staff, Students, Free, Doctors, Housewives, Retirees, Teachers, Students, Police,
Managers, Drivers, Workers, Children, Engineer, Journalist, Cameraman, and Ambassador, we put 1 to 17. For empty fields, we put 0 .

Features with very different values that don't have influence on data mining and features with very similar values with undesirable effect on model accuracy were omitted after investigation.

In the rest, satisfaction which is a basic variable of this research is classified based on C\&RT and CHAID algorithm.

\subsection{Modeling}

In this step, classification technique was employed to investigate effective factors on cardiac medical tourism in Iran. Three different methods were compared with each other as follows:

First method: Binary decision tree obtained from C\&RT method has the least error.

Second method: Non-binary decision tree obtained from CHAID algorithm has more errors than the binary method.

Third method: Multiple linear Regressions: It is a statistical prediction method that uses mathematical formula and calculations of independent variables effects on dependent variables that has negligible error level with research data.

A model which has less error or more precision is better evaluated. C\&RT model has less error level than linear regression model.

Related precision to C\&RT model is 0.922 and linear regression precision is 0.825 and CHAID decision tree precision is 0.084 .

\subsection{CHAID and C\&RT Algorithm}

2.6.1. C\&RT (Classification and Regression Tree) Algorithm The data obtained from C\&RT classification algorithms in Clementine software is shown in Figure 1.

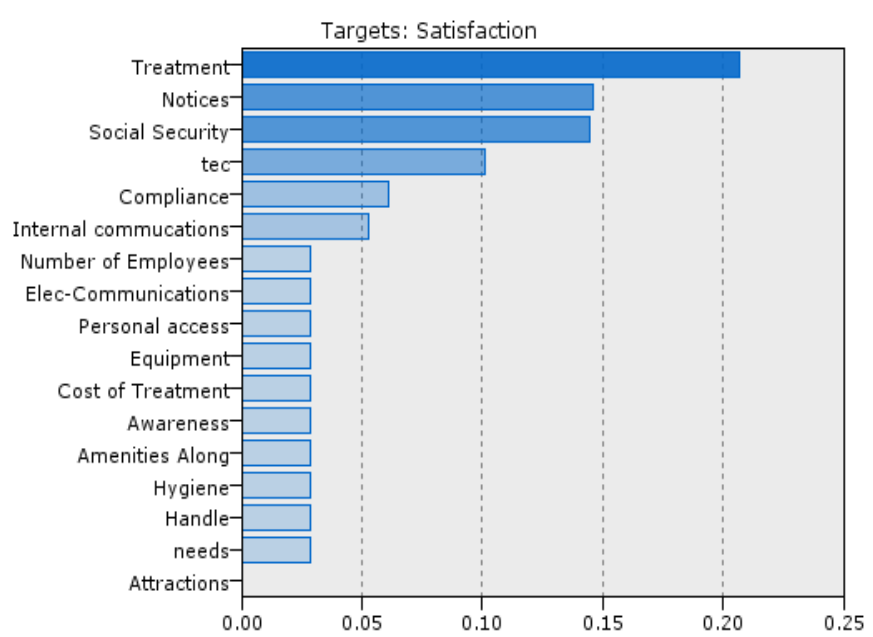

Figure 1. The variable impact on heart medical tourists' satisfaction algorithm using C \& RT

According to defined variables and obtained data from hospital management, questionnaire resulting from algorithm C \& RT, and characteristics of staff have the most effects on medical tourists' satisfaction. Then, social security feature was obtained as the second factor. Third, appropriate information in various media has the most effects in tourism satisfaction and increases and features such as regulation, physicians proper relationships with patients, availability of personnel, health and other needs of the patient, number of employees, equipment, personnel knowledge, appropriateness of costs, electronic communication, handling of the patient and considering the possibility for companion are in the next order. Tree diagram of the used model in this research is shown in Figure 2.

By clicking on the $(+)$ sign under the related rectangular to node 1, it is seen that 97 from 130 people were selected for behavior variable who selected smaller or similar numbers than $2.5,1$, and 2 . Other 33 people selected choices 3,4 , and 5.

2.6.2. CHAID (Chi-Squared Automatic Interaction Detector) Algorithm

Decision tree obtained from CHAID shows that variable accurate information, social security, and quality are effective variables. According to predicted criterion, effect of independent variable in objective variable, indicates that each tree level is processes and one variable is selected. 


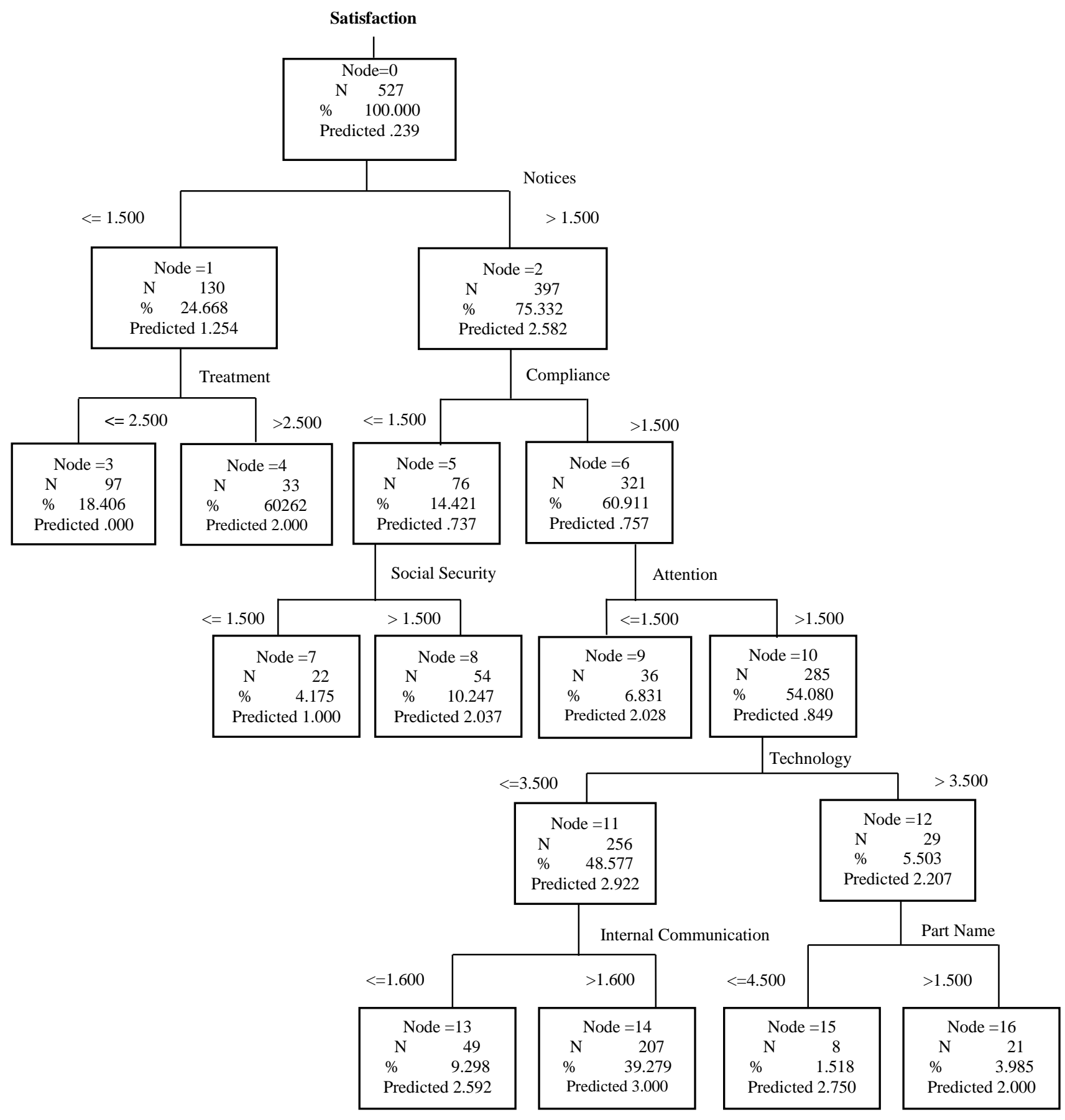

Figure 2. Tree diagram related to data used in Clementine model

As can be seen in the tree, we have 16 nodes, their initial shape are shown for more transparency. The tree diagram of the used model in this research is shown in Figure 3.

The first node is related to patient satisfaction which encompasses total records, $N$ represents number of people that belonged to the current node and percentage means of people. By clicking on the plus sign $(+)$ under the rectangle of the node, Clementine model can be seen.

The mentioned diagram means that $24.668 \%$ of all people chose 1 for informing variable which means "very little" and others, 397 people $(75.332 \%)$, chose 2 to 5 . It means they selected little, medium, high, very high.

\subsection{Measuring Precision in Decision Tree}

CART (Classification and Regression Tree) method makes a decision tree with binary divisions. In this method, Gini index is introduced as a criterion of selecting proper variables. Gini index is a common division index adopted from Corrado Gini, the Italian economist and statistician. This measurement used by biologists and ecologists who need to study population distribution shows probability of selecting 2 cases of similar population which were randomly selected. Or a net population, one probability is 1 . A fully net node will have Gini score. A balance node has 0.5 Gini score. Gini score is calculated by the following formula:

$$
\operatorname{GINI}(t)=1-\sum_{j}[p(j \mid t)]^{2}
$$

In the mentioned relationship, relative frequency of the $j^{\text {th }}$ class is in node $t$. Gini index diagram for the big nodes have 1 showing net degree of decision tree. 


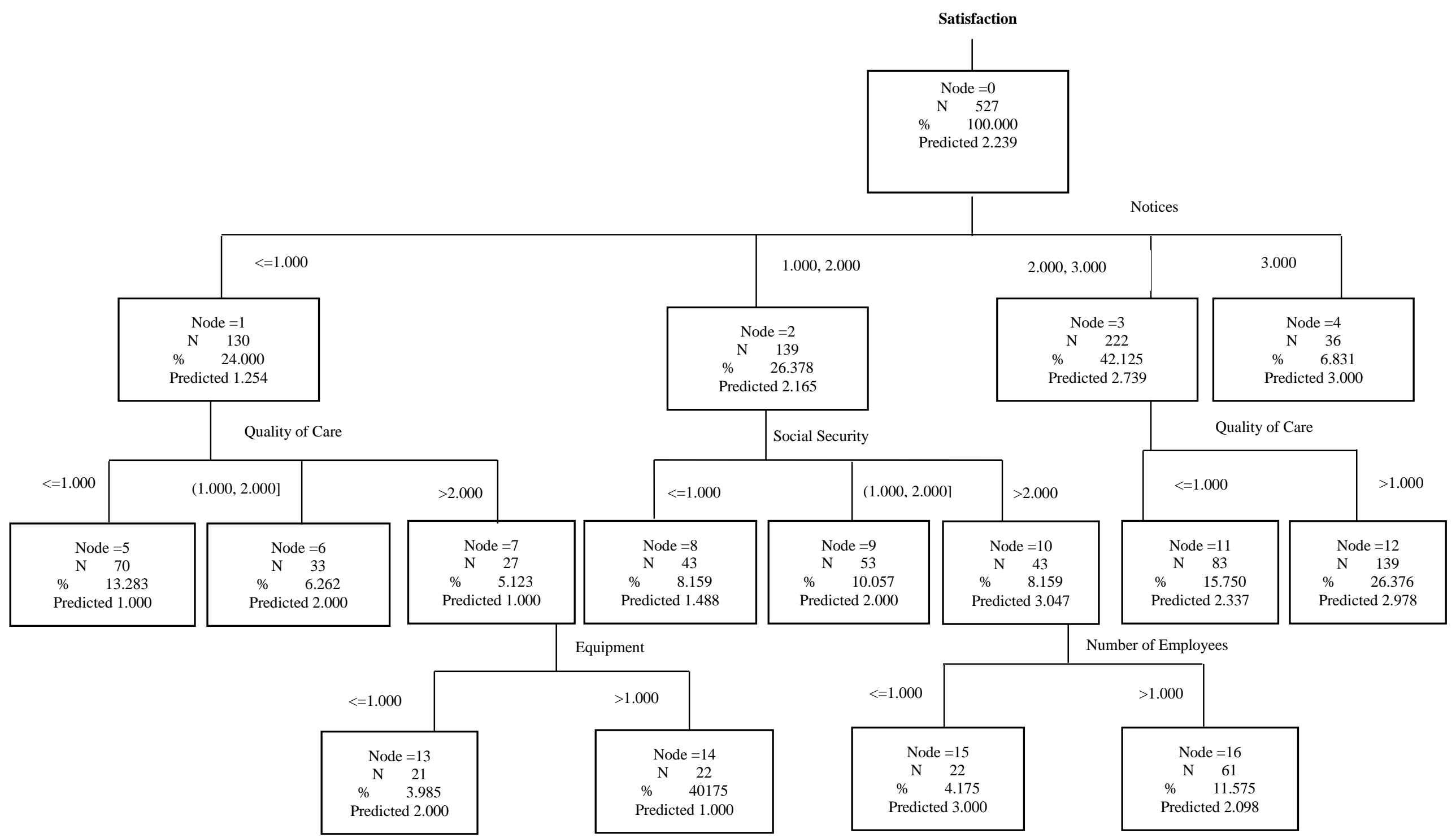

Figure 3. A scheme of CHAID Algorithm in Clementine 


\subsection{Multiple Linear Regression}

\subsubsection{Suggested Research Model and Hypotheses}

According to expected purposes and results, the main hypothesis of research as relational non-direction (twotailed) hypothesis includes the relationship between the mentioned factors simultaneously and Iran heart medical tourism. In addition, secondary hypothesis includes the relationship between independent and dependent variables.

\subsubsection{Main Hypotheses of Linear Regression}

Investigating Spearman correlation and significant relationship among dependent and independent variables.

Pearson correlation is used for ordinal variables, the results obtained are shown in Table 1.

Table 1. Pearson correlation

\begin{tabular}{lcc}
\hline Variable & The Correlation Coefficient Sig.(2-tailed) \\
\hline Equipment & $0.466^{* *}$ & 0.000 \\
\hline Technology & $0.360^{* *}$ & 0.000 \\
\hline Quality of care & $0.235^{* *}$ & 0.000 \\
\hline Knowledge & $0.305^{* *}$ & 0.000 \\
\hline The cost of treatment & $0.391^{* *}$ & 0.000 \\
\hline Investigation & $0.463^{* *}$ & 0.000 \\
\hline Notices & $0.746^{* *}$ & 0.000 \\
\hline Needs & $0.347^{* *}$ & 0.000 \\
\hline Employee behavior & $0.371^{* *}$ & 0.000 \\
\hline Availability of personnel & $0.065^{* *}$ & 0.139 \\
\hline Good communication & $0.360^{* *}$ & 0.000 \\
\hline electronic banking & -0.001 & 0.989 \\
\hline Number of Employees & $0.224^{* *}$ & 0.000 \\
\hline Electronic communication & $100^{*}$ & 0.022 \\
\hline Social Security & $0.416^{* *}$ & 0.000 \\
\hline Quality & $0.367^{* *}$ & 0.000 \\
\hline Hygiene & -0.023 & 0.591 \\
\hline Tourist attractions & $0.364^{* *}$ & 0.000 \\
\hline Mobile facilities & $0.358^{* *}$ & 0.000 \\
\hline Regulations & $0.679^{* *}$ & 0.000 \\
\hline transport cost & $0.341^{* *}$ & 0.000 \\
\hline ** confidence level is significant & & \\
\hline 95\% confidence level is significant & & ${ }^{* *}$ \\
\hline
\end{tabular}

Table 2. Skewness and Kurtosis values for satisfaction variable

\begin{tabular}{lcc}
\hline Variable & Skewness & Elongation \\
\hline Satisfaction (the dependent variable) & -0.353 & -1.182
\end{tabular}

Dependent variable should have normal distribution.

Coefficient of skewness and kurtosis coefficient as 2 main indexes of data distribution are shown in Table 2.

1. Skewness: It is a criterion for symmetry or asymmetry of distribution function. Skewness is zero in fully symmetrical distribution.

2. Kurtosis shows height of a distribution. In other words, kurtosis is a criterion of curve height in maximum point. Kurtosis is always compared to the normal distribution kurtosis. Kurtosis value for a normal distribution is 3 .

3. Generally, the amount of skewness and kurtosis of the data is in the range of 2,2 .

\section{1. $R$ Square}

$\mathrm{R}$ or $\mathrm{R}^{2}$ square coefficient determines how much percentage of dependent variables changes are determined by independent variable which is 89.5 in this research.

\section{Significant}

In ANOVA, if significance is less than 0.05, equation is linear regression and if significance is more than 0.05 , equation is not linear regression.

3. Durbin-Watson test

No correlation among model errors: Durbin-Watson test. Histogram test results is shown in Figure 4.

$$
D W=\frac{\sum\left(e_{t}-e_{t-1}\right)^{2}}{\sum e_{t}^{2}}
$$

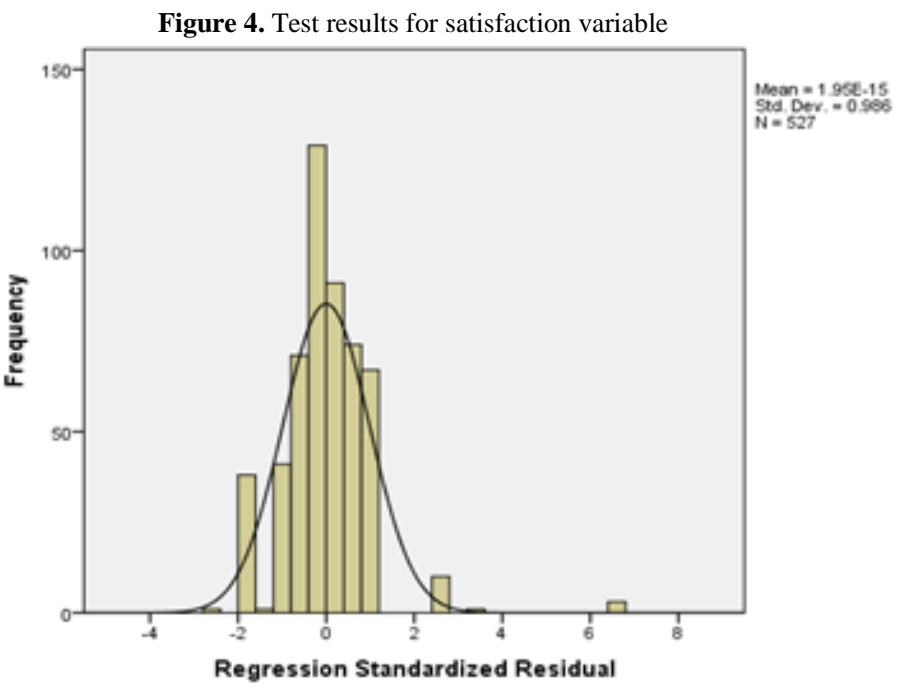

Resulting value and descriptions of test are added.

1. Error distribution should be normal.

2. Average (mathematical expectation) of errors should be zero.

3. Error variance should be constant.

\subsection{Collinear Test}

In multiple regression analysis where independent variables are self-correlated, there is internal or multiple linear correlation among them. The purpose is that correlation is an internal relationship among independent variables

Collinearity exists nearly in all regression models. "Total collinearity" defects occur if there is high correlation among independent variables. In spite of high $\mathrm{R}^{2}$, model doesn't have high validity.

\subsection{Collinear Cognition: Tolerance}

Tolerance is the change in ratio between 0 and 1 . Collinear probability VIF $>10$ and TOLERANCE $<2$

$$
\begin{aligned}
& \text { tolerence }=1-R_{j}^{2} \\
& V I F=\frac{1}{\text { tolerance }}
\end{aligned}
$$

\section{Results}

The greatest accuracy value (0.922), with respect to this algorithm, personnel behavior, social security and communication variables are respectively the most important factors for medical tourist attraction. The results obtained from this research indicate that C\&RT binary decision tree has the smallest error value (0.078) and the greatest accuracy value (0.922) in Table 3. With respect to this algorithm, personnel behavior, social security and communication variables are respectively the most important factors for medical tourist attraction. 
In this research, first the factors affecting the heart medical tourism were determined through the review and study of previous articles and help of expert's opinions. Then, using C\&RT, CHAID and Multiple Linear Regression methods and Clementin software, ranking and comparison of variables were performed.

\subsection{Investigating the Relationship among Independent and Dependent variables of Research by Multiple Linear Regression}

Simultaneous effect of independent variables on dependent variables of research was investigated by multiple linear regression and stepwise method.

As seen in Table 4, significance for proper facilities, using daily technology, and standard, therapy quality, adequate awareness and knowledge, full and timely preparation of rooms, medical (heart) tourism in various media, quick and timely satisfaction of needs of patients and his companions, easy access to need, physicians abilities in making proper relationships, adequate number of staff, presence of social security, medical services quality being effective, satisfaction from hospital hygiene, presence of tourist attractions, considering laws and regulations, and proper transportation cost are less than 0.05 showing linear relationship among the mentioned factors and satisfaction from Iran medical tourism. On the other hand, significance was more than 0.05 for variables of price being logical and medical costs, e-banking, e-relationship, and facilitation for patient companions which indicates on-existence of linear relationship among the mentioned factors and satisfaction from Iran hearth medical tourism. Determination coefficient $\left(\mathrm{R}^{2}\right)$ was 0.941 . Since variables of medical costs and facilitations break linear regression hypotheses, natural algorithm method were used instead of numbers, deleted figures and final results and line equation are shown in Table 4 and Table 5.
Tolerance $=0.059<0.2$

VIF $=16.846>10$

$$
\begin{aligned}
& \cdots \\
& \mathrm{Sig}=0.540>0.05 \\
& \llcorner\cdots-\cdots-\cdots-\cdots-\cdots-\cdots
\end{aligned}
$$

\subsection{Multiple Linear Regression Equation}

Satisfaction $=-2.431+0.210$ Informing +0.078 Regulations +-0.083 Behavior +0.310 Physicians' relationships +-0.121 Hygiene +0.360 Installations +0.353 Tourists Attractions +0.260 Satisfying needs +0.268 Quality + 0.120 Staff accessibility + 0.184 Technology + 0.149 Companion' facilitations +-0.088 Transportation costs +0.076 Security +0.067 Staff numbers

\section{Discussion}

In this research, the relevant variables for medical tourism in the field of cardiovascular surgeries were first extracted. On clinical examination [18], research obtained variables in this study were not extracted and analyzed; but a native expert localized it and data collection was made by surveys, and hospital statistical information were accessed where the records of medical tourists have reached a total number of 640. Then 526 correct records were extracted by preprocessing of data. Clementine software and categorization tree were applied to the data. This categorization was made utilizing regression method in descriptive statistics where it was shown that the categorization tree is more useful. Then the driven tree was studied using the tree evaluation criteria and it was shown that the decision tree in this result shows a better purity grade which is acceptable. According to the research findings, the most important propositions that could be considered as a benchmark to foster the heart medical tourism industry in Iran include education and improvement of organizational culture regarding different people from different cultures and nationalities.

Table 3. Ranking and comparison of variables

\begin{tabular}{|c|c|c|c|c|}
\hline Key Variables & Accuracy & Error Value & Type & Algorithm \\
\hline Behavior, security, Informing & 0.922 & 0.078 & Recursive Binary Decision Tree & C\&RT \\
\hline Quality, security, Informing & 0.898 & 0.084 & Non-binary decision tree & CHAID \\
\hline Informing, Regulations, Behavior & 0.895 & 0.984 & Statistical Calculations & Multiple Linear Regression \\
\hline
\end{tabular}

Table 4. Test results of multiple regression model between independent variables and the satisfaction of heart medical tourism in Iran

\begin{tabular}{|c|c|c|c|c|c|c|c|}
\hline Independent Variable & Sig. & $\begin{array}{r}\text { B Coefficient } \\
\text { Constant }=\mathbf{- 1 . 8 1 7}\end{array}$ & VIF & Tolerance & $\mathbf{R}^{2}$ & DW & $\mathbf{F}$ \\
\hline Installation & 0.000 & 0.246 & 3.398 & 0.294 & \multirow{19}{*}{0.941} & \multirow{19}{*}{2.100} & \multirow{19}{*}{478.114} \\
\hline Technology & 0.000 & -0.184 & 2.528 & 0.396 & & & \\
\hline Medical quality & 0.000 & 0.443 & 7.023 & 0.142 & & & \\
\hline Awareness & 0.000 & 0.140 & 5.084 & 0.197 & & & \\
\hline Medical costs & 0.453 & 0 & 3.820 & 0.262 & & & \\
\hline Consideration & 0.000 & 0.381 & 2.960 & 0.338 & & & \\
\hline Informing & 0.000 & 0.381 & 2.960 & 0.338 & & & \\
\hline Staff behavior & 0.330 & 0 & 5.031 & 0.199 & & & \\
\hline Staff access & 0.000 & 0.461 & 4.051 & 0.247 & & & \\
\hline Physicians' relationships & 0.000 & 0.139 & 3.445 & 0.290 & & & \\
\hline Electronic banking & 0.540 & -0.919 & 16.846 & 0.059 & & & \\
\hline Staff numbers & 0.000 & 0.082 & 4.095 & 0.244 & & & \\
\hline Electronic relationships & 0.806 & 0 & 8.176 & 0.122 & & & \\
\hline Social security & 0.000 & 0.336 & 4.983 & 0.201 & & & \\
\hline Quality & 0.000 & 0.159 & 2.097 & 0.477 & & & \\
\hline Tourists attractions & 0.000 & 0.525 & 2.596 & 0.385 & & & \\
\hline Companion facilities & 0.744 & 0 & 4.385 & 0.228 & & & \\
\hline Considerations & 0.000 & -0.175 & 6.233 & 0.160 & & & \\
\hline Transportation cost & 0.000 & 0.142 & 3.116 & 0.321 & & & \\
\hline
\end{tabular}


Noori F et al. Iran's Medical Tourism Development Model

Final Test Results of Multiple Linear Regressions

Table 5. The Final Test Results of Multiples Linear Regression of heart medical tourism in Iran

\begin{tabular}{|c|c|c|c|c|c|c|c|}
\hline Independent variable & Sig. & $\begin{array}{c}\text { B coefficient } \\
\text { Constant }=2.431\end{array}$ & VIF & Tolerance & $\mathbf{R 2}$ & DW & $\mathbf{F}$ \\
\hline Installation & 0.000 & 0.360 & 3.408 & & \multirow{18}{*}{0.293} & \multirow{18}{*}{0.895} & \multirow{18}{*}{289.975} \\
\hline Technology & 0.000 & -0.184 & 1.696 & 0.589 & & & \\
\hline Medical quality & 0.181 & 0 & 3.016 & 0.332 & & & \\
\hline Awareness & 0.656 & 0 & 4.930 & 0.343 & & & \\
\hline Medical costs & 0.564 & 0 & 2.916 & 0.343 & & & \\
\hline Consideration & 0.249 & 0 & 2.319 & 0.431 & & & \\
\hline Informing & 0.000 & 0.210 & 3.925 & 0.255 & & & \\
\hline Staff behavior & 0.001 & -0.83 & 3.179 & 0.315 & & & \\
\hline Staff access & 0.000 & 0.120 & 2.969 & 0.337 & & & \\
\hline Physicians' relationships & 0.000 & 0.310 & 2.050 & 0.488 & & & \\
\hline Staff numbers & 0.001 & 0.067 & 3.039 & 0.329 & & & \\
\hline Electronic relationships & 0.265 & 0 & 7.993 & 0.125 & & & \\
\hline Social security & 0.001 & 0.076 & 2.009 & 0.498 & & & \\
\hline Quality & 0.000 & 0.268 & 2.112 & 0.474 & & & \\
\hline Hygiene & 0.000 & -0.121 & 1.984 & 0.504 & & & \\
\hline Companion facilities & .000 & 0.149 & 2.803 & 0.357 & & & \\
\hline Considerations & 0.000 & 0.078 & 3.495 & 0.286 & & & \\
\hline Transportation cost & 0.000 & -0.088 & 1.946 & 0.514 & & & \\
\hline
\end{tabular}

Table 6. Comparison of obtained results from research algorithms

\begin{tabular}{lccc}
\hline Algorithm & Algorithm Type & Error Level & Main Variables \\
\hline C\&RT & Reversible binary decision tree & 0.078 & Behavior, security, informing \\
CHAID & Non-binary decision tree & 0.084 & Informing, security, quality \\
Multiple linear regression & Statistical calculations & 0.895 & Informing, regulation, behavior \\
\hline
\end{tabular}

\section{Conclusion}

In this research, effective factors on heart medical tourism were investigated with the help of experts in this field; thereafter, C\&RT and CHASID models by Clementine software and multiple linear regression variables were compared and ranked as shown in Table 6.

\section{Acknowledgments}

This article was extracted from a medical services management master's thesis.

\section{Authors' Contributions}

All authors contributed equally in the preparation of this paper.

\section{Financial Disclosure}

The authors declare they have no financial disclosure.

\section{Funding/Support}

This research was supported by the Tarbiat Modares University, Tehran, Iran.

\section{References}

1. Ghanbari S, Hajinejad A, Rahmani P. Formulating strategic plan of medical tourism development. Int $\mathrm{J}$ Travel Med Glob Health. 2014;2(4):149-54.

2. Ayoubian A, Tourani S, Dehaghi ZH. Medical tourism attraction of Tehran hospitals. Int J Travel Med Glob Health. 2014;1(2):95-8.

3. Hosseini SM, Maher A, Ayoubian A, Tehrani DS, Amini-Anabad H, Dehaghi ZH. Development strategy of health tourism in Iran. Int J Travel Med Glob Health. 2015;3(4).

4. Izadi M, Ayoobian A, Nasiri T, Joneidi N, Fazel M, Hosseinpourfard M. Situation of health tourism in Iran; opportunity or threat. Mil Med J. 2012;14(2):69-75.

5. Jafari M, Bahador M, Ravangard R. Health tourism: opportunities, constraints, obstacles and solutions. Int J Travel Med Glob Health. 2016;4(1):37-8. doi: 10.20286/ijtmgh-040137
6. Pan T-J, Chen W-C. Chinese medical tourists-Their perceptions of Taiwan. Tourism Manag. 2014;44:108-12. doi: 10.1016/j.tourman.2014.02.008

7. Delgoshaei B, Ravaghi H, Abolhassani N. Importance-performance analysis of medical tourism in Iran from medical tourists and medical services provider's perspective: 2011. Middle East J Sci Res. 2012;12(11):1541-7.

8. Izadi M, Saadat SH, Ayoubian A, Dehaghi ZH, Karbasi MR, Jalali AR. Health tourism in Iran; identifying obstacles for development of this industry. Int $\mathbf{J}$ Travel Med Glob Health. 2014;1(2):89-94.

9. Goeldner CR, Ritchie JB. Tourism: Principles, practices, philosophies: John Wiley \& Sons; 2006.

10. Kass GV. An exploratory technique for investigating large quantities of categorical data. Appl Stat. 1980:119-27. doi: 10.2307/2986296

11. Pourkhaghan Z, Faez SEP, Pourkhaghan S, Ghahrieh S. Interaction of economic indicators and medical tourism industry. Int J Travel Med Glob Health. 2014;1(3):133-9.

12. Memish ZA, Al-Tawfiq JA, Assiri A, Mohammed M, Bamgboye EA, Alhakeem R. Epidemiology of mumps and rubella in the Kingdom of Saudi Arabia: 2009-2011-Implications for immigration and travel. Travel Med Infect Dis. 2015;13(3):261-2. doi: 10.1016/j.tmaid.2015.04.002

13. Al-Abri SS, Abdel-Hady DM, Al-Abaidani IS. Knowledge, attitudes, and practices regarding travel health among Muscat International Airport travelers in Oman: Identifying the gaps and addressing the challenges. J Epidemiol Glob Health. 2016;6(2):67-75. doi: 10.1016/j.jegh.2016.02.003

14. Anari PY, Vaghefi M, Sepehri M, Tehrani DS, Habibi M. Travel medicine: a systematic review. Int $\mathrm{J}$ Travel Med Glob Health. 2015;3(3):109-11. doi: 10.20286/ijtmgh-0303109

15. Han H, Hyun SS. Customer retention in the medical tourism industry: Impact of quality, satisfaction, trust, and price reasonableness. Tourism Manag. 2015;46:20-9. doi: 10.1016/j.tourman.2014.06.003

16. Jabbari A, Agharahimi Z, Moradi Zohreh S, Ferdosi M. Potentials of cosmetic surgery tourism in Isfahan: a qualitative study. Health Inf Manag. 2012;9(5):686-96.

17. Tourani S, Tabibi SJ, Tofighi S, Shaarbafchi Zadeh N. Medical tourism in Iran: analysis of opportunities and challenges with MADM approach. Res J Biol Sci. 2010;5(3):251-57. doi: 10.3923/rjbsci.2010.251.257

18. Akdag H, Kalaycı T, Karagöz S, Zülfikar H, Giz D. The evaluation of hospital service quality by fuzzy MCDM. Appl Soft Comput. 2014;23:239-48. doi: 10.1016/j.asoc.2014.06.033 